\title{
Volumetric modulated arc therapy for lung stereotactic radiation therapy can achieve high local control rates
}

Hideomi Yamashita*, Akihiro Haga, Wataru Takahashi, Ryousuke Takenaka, Toshikazu Imae, Shigeharu Takenaka and Keiichi Nakagawa

\begin{abstract}
Purpose: The aim of this study was to report the outcome of primary or metastatic lung cancer patients undergoing volumetric modulated arc therapy for stereotactic body radiation therapy (VMAT-SBRT).

Methods and materials: From October 2010 to December 2013, consecutive 67 lung cancer patients received single-arc VMAT-SBRT using an Elekta-synergy system. All patients were treated with an abdominal compressor. The gross tumor volumes were contoured on 10 respiratory phases computed tomography (CT) datasets from 4-dimensional (4D) CT and merged into internal target volumes (ITVs). The planning target volume (PTV) margin was isotropically taken as $5 \mathrm{~mm}$. Treatment was performed with a D95 prescription of 50 Gy (43 cases) or 55 Gy (12 cases) in 4 fractions for peripheral tumor or 56 Gy in 7 fractions (12 cases) for central tumor.
\end{abstract}

Results: Among the 67 patients, the median age was 73 years (range, 59-95 years). Of the patients, male was $72 \%$ and female $28 \%$. The median Karnofsky performance status was $90-100 \%$ in 39 cases (58\%) and $80-90 \%$ in 20 cases (30\%). The median follow-up was 267 days (range, 40-1162 days). Tissue diagnosis was performed in 41 patients (61\%). There were T1 primary lung tumor in 42 patients (T1a in 28 patients, T1 b in 14 patients), T2 in 6 patients, three T3 in 3 patients, and metastatic lung tumor in 16 patients. The median mean lung dose was 6.87 Gy (range, 2.5-15 Gy). Six patients (9\%) developed radiation pneumonitis required by steroid administration. Actuarial local control rate were $100 \%$ and $100 \%$ at 1 year, $92 \%$ and $75 \%$ at 2 years, and $92 \%$ and $75 \%$ at 3 years in primary and metastatic lung cancer, respectively ( $p=0.59$ ). Overall survival rate was $83 \%$ and $84 \%$ at 1 year, $76 \%$ and $53 \%$ at 2 years, and $46 \%$ and $20 \%$ at 3 years in primary and metastatic lung cancer, respectively $(p=0.12)$.

Conclusions: Use of VMAT-based delivery of SBRT in primary in metastatic lung tumors demonstrates high local control rates and low risk of normal tissue complications.

Keywords: Primary lung cancer, Metastatic lung cancer, Stereotactic body radiation therapy, IMRT, VMAT

\section{Introduction}

Stereotactic body radiation therapy (SBRT) is emerging as an effective treatment for patients with medically inoperable, early stage lung cancers as evidenced by local control rates reaching $90 \%$ at three years with relatively minimal toxicity $[1,2]$.

The report from Mayo Clinic to validate the use of SBRT of 50 Gy in 5 fractions using IMRT 7 non-coplanar beams for 26 patients with medically inoperable Stage I

\footnotetext{
* Correspondence: yamachan07291973@yahoo.co.jp

Department of Radiology, The University of Tokyo Hospital, 7-3-1 Hongo, Bunkyo-ku, Tokyo 113-8655, Japan
}

\section{Ciomed Central}

lung cancer was published [3]. Use of IMRT during SBRT has not been without issues for some authors, with questions on the feasibility of IMRT delivery within small fields typical of SBRT [4], and concerns that organ motion could negate the benefits of the IMRT [5]. Recently, it was reported that volumetric modulated arc therapy (VMAT), which is a novel rotational technique and an extension of intensity modulated radiation therapy (IMRT), is applicable for SBRT for lung tumors [6-8]. This technique achieves treatment plan qualities comparable to the noncoplanar IMRT technique and dramatically decreases the total treatment time for each fraction [9]. 
To date, there are no published clinical studies specially addressing VMAT during SBRT for lung cancer. The purpose of the present report is therefore to provide clinical results for a group of primary or metastatic lung cancer patients whom we have treated with a VMAT-SBRT approach. This study has been done retrospectively and was proven by a local ethic/IRB board (No. 3372).

\section{Materials and methods}

Tumor biopsy was attempted in all patients to confirm malignancy unless medically or surgically contraindicated. Before initiation of treatment, patients were expected to undergo complete history taking and physical examination, contrast-enhanced computed tomography (CT) imaging of the chest, $\left[{ }^{18} \mathrm{~F}\right]$ fluorodeoxyglucose positron emission tomography (FDG-PET) scanning for staging, pulmonary function testing (PFTs) including forced expiratory volume at $1 \mathrm{~s}$ (FEV1.0), and diffusion capacity to carbon monoxide (DLCO) only before SBRT and brain imaging (contrast-enhanced $\mathrm{CT}$ or magnetic resonance imaging [MRI]). The PFT was not performed routinely after SBRT.

\section{Patient and tumor characteristics}

From October 2010 to December 2013, consecutive 67 lung cancer patients received single-arc VMAT-SBRT using an Elekta-synergy system. Complete data were acquired and evaluated for all 67 patients. Patient and tumor characteristics are summarized in Table 1. Among the 67 patients, the median age was 73 years (range, 5995 years). Of the patients, male was $72 \%$ and female $28 \%$. The median Karnofsky performance status was $90-$ $100 \%$ in 39 cases (58\%) and $80-90 \%$ in 20 cases $(30 \%)$. Tissue diagnosis was performed in 41 patients (61\%). There were $\mathrm{T} 1$ primary lung tumor in 42 patients ( $\mathrm{T} 1 \mathrm{a}$ in 28 patients, T1b in 14 patients), T2 in 6 patients, three T3 in 3 patients, and metastatic lung tumor in 16 patients. In our institution, some T3 tumors only with direct invasion to chest wall, without lymph node metastasis, and without the indication of radical surgery were treated with SBRT-alone. As to histology, squamous cell carcinoma was diagnosed in 26 cases, adenocarcinoma in 10 cases, small cell carcinoma in 2 cases, and nonsmall cell carcinoma, carcinoma, and undifferentiated carcinoma in one case, respectively.

$39 \%$ of the patients were treated without histology. In primary cases without histology, PET/CT scan was compulsory. Either the SUV-max value over 3.0 by PET scan or the obvious tendency for increased tumor size by CT scans in the absence of significant up-take increase of FDG was necessary conditions for SBRT. Such cases had been discussed in weekly interdisciplinary cancer board and treatment decisions were based on recommendations of such a cancer board.
Table 1 Patient and tumor characteristics

\begin{tabular}{|c|c|c|}
\hline Factors & No. & Rate \\
\hline \multicolumn{3}{|l|}{ Age } \\
\hline Range & 56-95 years old & \\
\hline Median & 73 years old & \\
\hline \multicolumn{3}{|l|}{ Sex } \\
\hline Male & 48 & $72 \%$ \\
\hline Female & 19 & $28 \%$ \\
\hline \multicolumn{3}{|l|}{ K-PS } \\
\hline $90-100 \%$ & 39 & $58 \%$ \\
\hline $80-90 \%$ & 20 & $30 \%$ \\
\hline $70 \%$ & 2 & $3 \%$ \\
\hline Unknown & 6 & $9 \%$ \\
\hline \multicolumn{3}{|c|}{ Smoking history } \\
\hline With & 29 & $43 \%$ \\
\hline Without & 17 & $25 \%$ \\
\hline Unknown & 21 & $31 \%$ \\
\hline \multicolumn{3}{|l|}{ T stage } \\
\hline T1a & 28 & $42 \%$ \\
\hline T1b & 14 & $21 \%$ \\
\hline $\mathrm{T} 2$ & 6 & $9 \%$ \\
\hline T3 & 3 & $4 \%$ \\
\hline Metastatic & 16 & $24 \%$ \\
\hline \multicolumn{3}{|l|}{$\begin{array}{l}\text { Pathological } \\
\text { confirmation }\end{array}$} \\
\hline Without & 26 & $39 \%$ \\
\hline With & 41 & $61 \%$ \\
\hline
\end{tabular}

Maximum diameter of

primary tumor

\begin{tabular}{|c|c|c|}
\hline Range & 7-51 mm & \\
\hline Median & $20 \mathrm{~mm}$ & \\
\hline \multicolumn{3}{|l|}{ Tumor location } \\
\hline Left upper & 18 & $27 \%$ \\
\hline Left lower & 10 & $15 \%$ \\
\hline Right upper & 17 & $25 \%$ \\
\hline Right middle & 9 & $13 \%$ \\
\hline Right lower & 13 & $19 \%$ \\
\hline \multicolumn{3}{|l|}{$\begin{array}{l}\text { Combined } \\
\text { chemotherapy }\end{array}$} \\
\hline $\begin{array}{l}\text { Both before and } \\
\text { after SBRT }\end{array}$ & 2 & $3 \%$ \\
\hline Before SBRT & 5 & $7 \%$ \\
\hline After SBRT & 3 & $4 \%$ \\
\hline None & 57 & $85 \%$ \\
\hline
\end{tabular}

Selection of these patients for SBRT was not limited by baseline performance, pulmonary status, or tumor location. Patients were followed-up initially 6 to 8 weeks 
after completion of SBRT, with same-day PFTs and contrast-enhanced CT imaging of the chest. Thereafter, routine follow-up was every 3 months for 2 years, with CT imaging at each visit and PFTs twice yearly. Tumor responses were classified into complete response, partial response, stable disease, and progression based on Response Evaluation Criteria in Solid Tumors (RECIST). Toxicity was assessed retrospectively from patient clinical data using the Common Terminology Criteria for Adverse Events version 4.0 (CTCAE).

All patients underwent computed tomography (CT)based SBRT planning for IMRT. Four-dimensional CT images for treatment planning were acquired with 2mm-thick slices using an Aquilion LB model scanner (16-slice; Toshiba). The patients were in the supine position and fitted with an abdominal compressor [10,11]. The Elekta stereotactic body frame (SBF) was also used to minimize breathing artifacts for treatment planning CT. Scans were performed using the AZ-733 V system (Anzai Medical) as an external respiratory monitoring system. Each respiratory phase scan was transferred to a Pinnacle ${ }^{3}$, version 9.0, system (Philips).

The gross tumor volume (GTV) was delineated using the lung window (window, $1600 \mathrm{HU}$; level, $-300 \mathrm{HU}$ ), on the 10 respiratory phase CT datasets from 4D CT respiratory sorting. Then, the ITV was produced using an integration of the GTVs as defined in these 10 phases. In all cases, the PTV was defined by adding a uniform 5$\mathrm{mm}$ margin to the ITV to compensate for setup errors. Critical structures including but not limited to ipsilateral and contralateral lungs, spinal cord, and esophagus were outlined. PET-CT data were not used directly for imageguided planning.

Patients received a D95 prescription of 50 Gy (43 cases) before August 2013 or 55 Gy (12 cases) after August 2013 in 4 fractions for peripheral tumor or 56 Gy in 7 fractions (12 cases) for central tumor as defined by RTOG 0236 [12]. The single-arc VMAT-SBRT with $6 \mathrm{MV}$ was created by SmartArc (Pinnacle ${ }^{3}$; Philips). Dose constraints for normal organs at risk for complications were the ipsilateral lung volume receiving $20 \mathrm{~Gy}$ (V20) $<10 \%$ and 5 Gy $<25 \%$ contralateral lung volume receiving 20 Gy (V20) $<0 \%$ and 5 Gy $<15 \%$; spinal cord volumes receiving 15 Gy (V15) $<0 \%$; heart volumes receiving 30 Gy $<0 \%$; liver volume receiving $30 \mathrm{~Gy}<0 \%$; body receiving $50 \mathrm{~Gy}<0 \%$. Dosimetric planning and plan analysis were performed in Pinnacle ${ }^{3}$. The collapsed cone convolution method (which was comparable to superposition method) in Pinnacle $^{3}$ was used as the heterogeneous correction method for the lungs. All final calculations were performed with a grid size of $2.0 \mathrm{~mm}$. Dose distributions were calculated using peak exhalation CT data.

In primary cases, SBRT was the only treatment modality and no additional chemotherapy treatment was performed during disease control. In metastatic cases, no patients had received lung irradiation before SBRT.

Recently, a system for performing pre-treatment respiration-correlated $\mathrm{CBCT}$, namely $4 \mathrm{D} \mathrm{CBCT}$, was developed by using an image-based recognition technique of the respiration phase $[9,13]$. Registration was semi-automatically performed using such pre-4D CBCT.

\section{Image guided RT procedure}

VMAT-SBRT was performed using a photon beam provided by a Synergy linear accelerator (Elekta) equipped with the $\mathrm{kV} \mathrm{CBCT}$ system that included a $\mathrm{kV}$-ray tube and flat-panel detector mounted on each side of the gantry, perpendicular to the treatment beam. In advance, the isocenter information of the treatment plan, regions of interest, and CT image set for peak exhalation were sent to the workstation with application software loaded onto the $\mathrm{x}$-ray volume imaging functionality PC (XVI system, version 4.2). As an image guided RT (IGRT) procedure, pre-4D CBCT images were acquired with $\mathrm{kV}$ imaging parameters of a beam of $120 \mathrm{kVp}$ and $20 \mathrm{~mA} /$ $20 \mathrm{~ms}$ at an axial field length of $20 \mathrm{~cm}$ with a bow-tie filter immediately before daily treatment. In this case, the typical number of frames was approximately 650 in a pre-CBCT scan. Tumor registration was performed between obtaining a planning CT image for peak exhalation and obtaining the aforementioned $4 \mathrm{D}$ CBCT image. In the registration procedure, the chamfer matching (bone matching) was used first, and then, the manual registration was performed using ITV and PTV. Thereafter, the patient couch was adjusted according to the registration result.

\section{Statistical analysis}

The outcomes of interest were local control and survival, measured from the time of the first day of SBRT until death or last patient contact. Local failure was defined as progressive and increasing CT scan abnormalities confirmed by progressive and incremental increases in the standardized uptake values (SUVs) of a lesion on serial PET imaging, with or without biopsy [14]. The survival probability was illustrated using Kaplan-Meier curves, and the log-rank test was used to determine whether a statistically significant difference was present among patient groups. The correlation coefficient was calculated between the occurrence of radiation pneumonitis required by steroid administration and the value of FEV1.0 or DLCO. The statistical analyses were performed using StatView 5.0 (SAS Institute, Cary, NC), and a value of $\mathrm{p}<0.05$ was considered statistically significant. 


\section{Results}

\section{Results of SBRT planning}

The median total treatment period was 6 days (range, 4-13 days). The ITV ranged from 1.6 to $80.1 \mathrm{cc}$ (median, $5.8 \mathrm{cc}$ ). The median maximum, minimum, and mean dose within PTV was 58 Gy (range, 52.7-77 Gy), 48 Gy (range, 10.660.7 Gy), and 53.9 Gy (50.6-68 Gy). The median mean lung dose was 6.87 Gy (range, 2.5-15 Gy). The median lung V20, V15, and V5 were 9\% (range, 3-21\%), 12\% (range, 5-30\%), and $25 \%(9-60 \%)$, respectively.

\section{Clinical results}

The median follow-up was 8.9 months (range, 1.338.7 months). Limiting to surviving cases, the minimum follow-up time was 10.1 months. At the time of analysis, 48 cases were alive and 17 cases were dead. Actuarial local control rate were $100 \%$ and $100 \%$ at 1 year, $92 \%$ and $75 \%$ at 2 years, and $92 \%$ and $75 \%$ at 3 years in primary and metastatic lung cancer, respectively ( $p=0.59$ ) (Figure 1). Overall survival rate was $83 \%$ and $84 \%$ at 1 year, $76 \%$ and $53 \%$ at 2 years, and $46 \%$ and $20 \%$ at 3 years in primary and metastatic lung cancer, respectively $(p=0.12)$ (Figure 2$)$. Actuarial disease control rate was $86 \%$ and $66 \%$ at 1 year, $78 \%$ and $41 \%$ at 2 years, and $78 \%$ and $41 \%$ at 3 years in primary and metastatic lung cancer, respectively ( $p=0.013)$ (Figure 3 ).

For the dead patients of primary 10 cases and metastatic 7 cases, the cause of death was other metastases but lung in 11 cases, local problems in one case, and other disease in 5 cases. All three cases with primary T3 tumor were dead of distant metastases. For living 7 cases with disease (primary 4 cases and metastatic 3 cases), the pattern of relapse was all distant metastases.

\section{Toxicity}

According to Common Terminology Criteria for Adverse Events (CTCAE) v4.0, grade 2 skin reactions were seen in

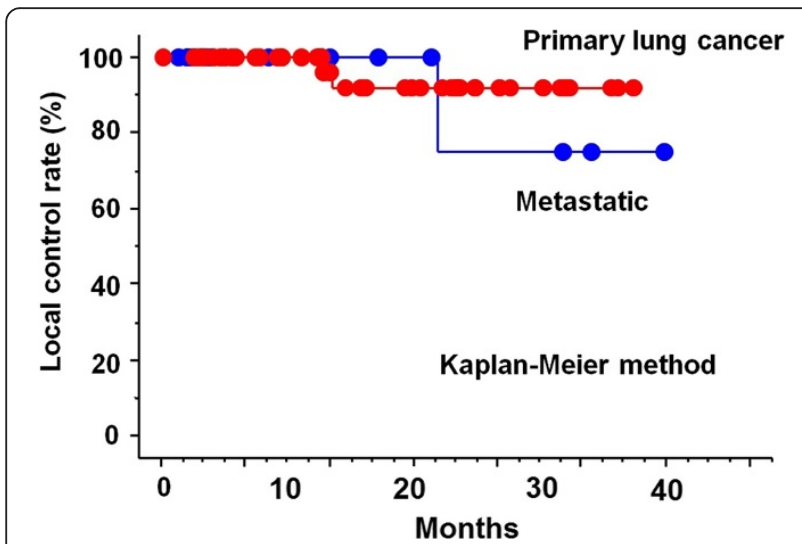

Figure 1 Local control curves by primary and metastatic lung cancer.

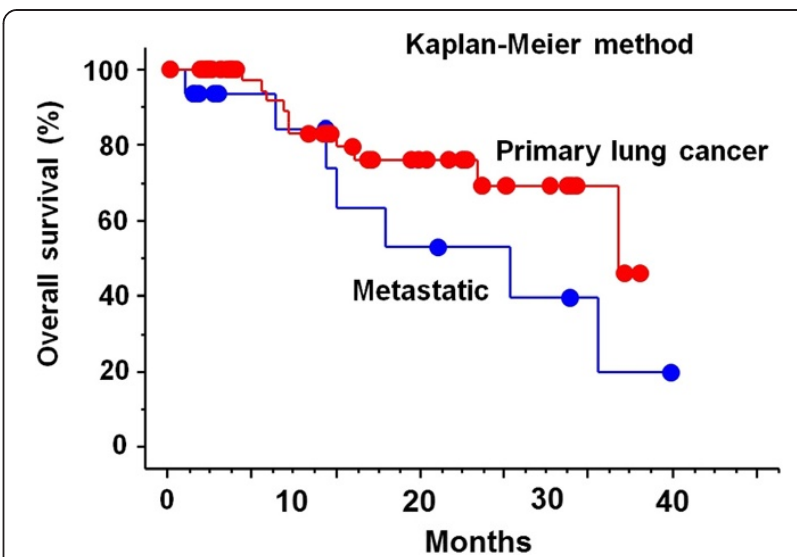

Figure 2 Overall survival curves by primary and metastatic lung cancer.

3 cases (grade 3 or more in zero) and grade 2 or more fatigue and chest wall pain requiring pain medication was never seen. Six patients (9\%) developed radiation pneumonitis required by steroid administration. There was no correlation between the occurrence of radiation pneumonitis required by steroid administration and the value of FEV1.0 or DLCO. No other toxicities not lower than grade 3 were seen. No rib fractures or late esophageal toxicities were seen.

\section{Discussion}

To our knowledge, this is the first report discussing the clinical result of IMRT SBRT using VMAT. One potential problem when inverse planning for lung cancer treatment is that the optimization of beam fluence only takes into account a single three-dimensional volumetric data set. For this, the actual VMAT that continuously delivers the dose in all respiratory phases may yield a dose distribution that differs from the plan. In order to compensate for this fact, the target volume can be

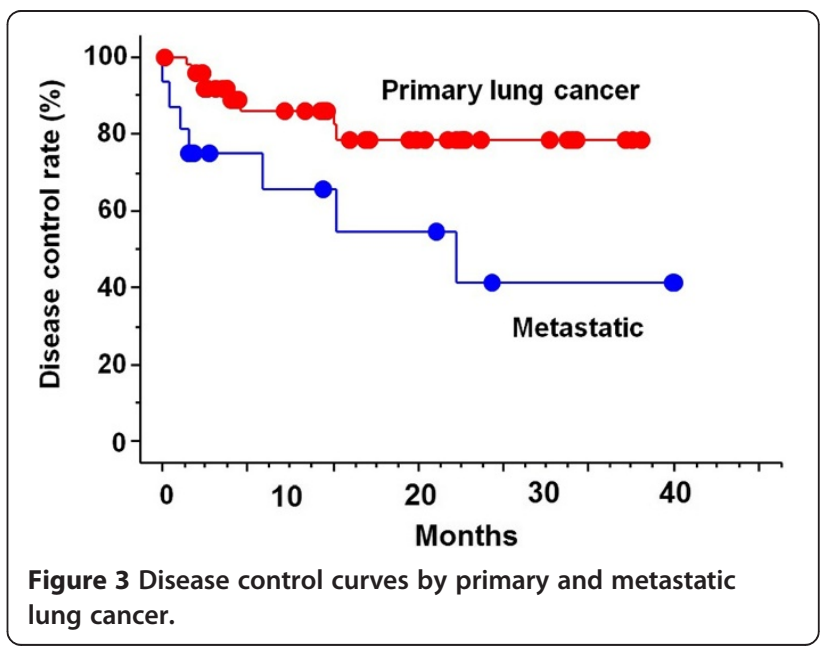


extended to encompass the range of target motion by using 4D-CT scan, and field shapes can be defined as the target surroundings. Alternatively, the inverse plan that constrains MLC motion in VMAT forms field shapes that do not hide the target in lung cancer treatment. In this case, the beam intensity is mainly modulated by changing gantry speed and dose rate. Recently, it was reported that VMAT, which is a novel rotational technique and an extension of IMRT, is applicable for SBRT for lung tumors $[7,15]$. This technique achieves treatment plan qualities comparable to the non-coplanar IMRT technique and dramatically decreases the total treatment time for each fraction. VMAT drastically reduced the amount of radiation intensity, known as the monitor unit (MU). Thus, VMAT was found to be able to deliver the desired dose distribution in a shorter time than IMRT. Nowadays, VMAT plays a main role in high-precision radiotherapy treatment.

The study by Crabtree et al. [16] compared prospective clinical trials of SBRT, sub-lobular resection, and radiofrequency ablation of three clinical trials and they concluded that, among appropriately matched patients, no difference was seen in early morbidity between sublobar resection and SBRT. Both articles by Andratschke et al. [17] and by Guckenberger et al. [18] presented data of a large number of patients with inoperable stage I non-small cell lung cancer concerning safety of treatment and patterns of failure. Andratschke et al. [17] reported that all 10 local failures were observed in patients with T2 tumors $(\mathrm{n}=61)$, isolated regional recurrence was observed in $7.6 \%(n=6)$, and the crude rate of distant progression was $20.7 \%(\mathrm{n}=22)$ and that no grade 3-5 acute toxicity was observed. Guckenberger et al. [18] reported that 3-year freedom from local progression was $79.6 \%$ and that radiation-induced pneumonitis grade 2 or higher was observed in 38 of 512 patients (7.4\%) and grade 5 was documented in two patients (0.4\%).

The present clinical results support VMAT-based IMRT-SBRT delivery for lung tumors. There have been previous nonclinical studies validating the appropriateness of IMRT in the setting of radiosurgery or SBRT. Benedict et al. [19] carried out a planning study on four brain small lesion cases to assess the potential for improved tumor coverage and normal tissue sparing using intensity-modulated stereotactic radiosurgery. These investigators demonstrated significant dosimetric improvements for the small, irregularly shaped lesions compared with the results of other techniques, with reductions in critical organ irradiation. Cardinale et al. [20] reported similar results favoring IMRT in their report on three SBRT techniques, namely, arcs, non-coplanar fixed fields, and IMRT. In an analysis of organ motion effects on IMRT treatments with segments of few monitor units. Seco et al. [21] concluded that for most clinical cases, any non-negligible effects of IMRT dose delivery may be clinically irrelevant when multiple beams are used. On the other hand, Dvorak et al. [22] studied the impact of IMRT and leaf width on SBRT for liver and lung lesions. They compared plans generated for 10 patients treated with multi-leaf collimator (MLC)-based conformal therapy to seven alternative plans including one for micro-MLC IMRT treatment as in the BrainLAB system. They found that no significant differences could be observed in terms of target conformity between standard and IMRT dose distributions. Videtic et al. [3] studied the use of SBRT of 50 Gy in 5 fractions using IMRT seven non-coplanar beams using a Novalis/BrainLAB system for medically inoperable 28 Stage I lung cancers of 26 patients. They reported that the 3-year local control for T1 (22 lesions) and T2 lesions (6 lesions) was $93.1 \%$ and $100 \%$, respectively. Hodge et al. [23] reported that delivery of IG-SBRT via helical tomotherapy using extreme hypofractionation in patients with early stage medically inoperable non-small cell lung cancer is feasible and well tolerated.

The limitations of this study include its retrospective nature. Although the small sample size also represents a limitation of this study, the median follow-up of close to 8.9 months, along with the meticulous documentation of patient status, lend support to the conclusions drawn from this cohort. Although this is a general limitation of lung cancer in following up with CT, it was the difficulty to discriminate between lung tissue changes after SBRT (i.e. post-RT scars) and true recurrences (i.e. progress) in this study, too.

\section{Conclusion}

Use of VMAT-based delivery of SBRT in primary in metastatic lung tumors demonstrates high local control rates and low risk of normal tissue complications.

\section{Competing interests}

The authors declare that they have no competing interests.

\section{Authors' contributions}

HY collected the clinical data, analysed it, and wrote this article. AK, WT, RT, $\mathrm{TI}$, and ST performed SBRT in a clinical setting. KN drafted the manuscript. All authors read and approved the final manuscript.

\section{Acknowledgments}

The authors kindly acknowledge (Reiko Kobayashi, Noriyasu Sekiya, Madoka Sakuramachi, Masataka Karube, Akihiro Nomoto, and Ryousuke Takenaka) for their support and assistance with data compilation.

Received: 16 April 2014 Accepted: 25 October 2014

Published online: 11 November 2014

\section{References}

1. Timmerman R, Paulus R, Galvin J, Michalski J, Straube W, Bradley J, Fakiris A, Bezjak A, Videtic G, Johnstone D, Fowler J, Gore E, Choy H: Stereotactic body radiation therapy for inoperable early stage lung cancer. JAMA 2010, 303:1070-1076.

2. Senthi S, Lagerwaard FJ, Haasbeek CJA, Senthi S, Lagerwaard FJ, Haasbeek CJ, Slotman BJ, Senan S: Patterns of disease recurrence after stereotactic 
radiotherapy for early stage non-small-cell lung cancer: a retrospective analysis. Lancet Oncol 2012, 13:802-809.

3. Videtic GM, Stephans K, Reddy C, Gajdos S, Kolar M, Clouser E, Djemil T: Intensity-modulated radiotherapy-based stereotactic body radiotherapy for medically inoperable early-stage lung cancer: excellent local control. Int J Radiat Oncol Biol Phys 2010, 77:344-349.

4. Jones AO, Das IJ: Comparison of inhomogeneity correction algorithms in small photon fields. Med Phys 2005, 32:766-776.

5. Bortfeld T, Jokivarsi K, Goitein M, Kung J, Jiang SB: Effects of intra-fraction motion on IMRT dose delivery: statistical analysis and simulation. Phys Med Biol 2002, 47:2203-2220.

6. Nakagawa K, Haga A, Shiraishi K, Yamashita H, Igaki H, Terahara A, Ohtomo K, Saegusa S, Shiraki T, Oritate T, Yoda K: First clinical cone-beam CT imaging during volumetric modulated arc therapy. Radiother Oncol 2009, 90:422-423.

7. Holt A, van Vliet-Vroegindeweij C, Mans A, Belderbos JS, Damen EM: Volumetric-modulated arc therapy for stereotactic body radiotherapy of lung tumors: a comparison with intensity-modulated radiotherapy techniques. Int J Radiat Oncol Biol Phys 2011, 81:1560-1567.

8. Deodato F, Cilla S, Macchia G, Caravatta L, Mignogna S, Massaccesi M, Picardi V, Digesu C, Sallustio G, Bonomo P, Piermattei A, Ferrandina G, Scambia G, Valentini V, Cellini N, Morganti AG: Extracranial radiosurgery with volumetric modulated arc therapy: feasibility evaluation of a phase I trial. Oncol Lett 2013, 5:1889-1896.

9. Takahashi W, Yamashita H, Kida S, Masutani Y, Sakumi A, Ohtomo K, Nakagawa K, Haga A: Verification of planning target volume settings in volumetric modulated arc therapy for stereotactic body radiation therapy by using in-treatment 4-dimensional cone beam computed tomography. Int J Radiat Oncol Biol Phys 2013, 86:426-431.

10. Yamashita H, Kobayashi-Shibata S, Terahara A, Okuma K, Haga A, Wakui R, Ohtomo K, Nakagawa K: Prescreening based on the presence of CT-scan abnormalities and biomarkers (KL-6 and SP-D) may reduce severe radiation pneumonitis after stereotactic radiotherapy. Radiat Oncol 2010, 5:32.

11. Yamashita H, Nakagawa K, Nakamura N, Koyanagi H, Tago M, Igaki H, Shiraishi K, Sasano N, Ohtomo K: Exceptionally high incidence of symptomatic grade $2-5$ radiation pneumonitis after stereotactic radiation therapy for lung tumors. Radiat Oncol 2007, 2:21.

12. Radiation Therapy Oncology Group. www.rtog.org. Last accessed August 13, 2009.

13. Kida S, Masutani Y, Yamashita H, Imae T, Matsuura T, Saotome N, Ohtomo K, Nakagawa K, Haga A: In-treatment 4D cone-beam CT with image-based respiratory phase recognition. Radiol Phys Technol 2012, 5:138-147.

14. Timmerman R, Papiez L, McGarry R, Timmerman R, Papiez L, McGarry R, Likes L, DesRosiers C, Frost S, Williams M: Extracranial stereotactic radioablation: results of a phase I study in medically noperable stage I non-small cell lung cancer. Chest 2003, 124:1946-1955.

15. McGrath SD, Matuszak MM, Yan D, McGrath SD, Matuszak MM, Yan D, Kestin LL, Martinez AA, Grills IS: Volumetric modulated arc therapy for delivery of hypofractionated stereotactic lung radiotherapy: a dosimetric and treatment efficiency analysis. Radiother Oncol 2009, 90:422-423.

16. Crabtree T, Puri V, Timmerman R, Fernando H, Bradley J, Decker PA, Paulus R, Putnum JB Jr, Dupuy DE, Meyers B: Treatment of stage I lung cancer in high-risk and inoperable patients: comparison of prospective clinical trials using stereotactic body radiotherapy (RTOG 0236), sublobar resection (ACOSOG Z4032), and radiofrequency ablation (ACOSOG Z4033). J Thorac Cardiovasc Surg 2013, 145:692-699.

17. Andratschke N, Zimmermann F, Boehm E, Schill S, Schoenknecht C, Thamm R, Molls M, Nieder C, Geinitz H: Stereotactic radiotherapy of histologically proven inoperable stage I non-small cell lung cancer: patterns of failure. Radiother Oncol 2011, 101:245-249.

18. Guckenberger M, Allgäuer M, Appold S, Dieckmann K, Ernst I, Ganswindt U, Holy R, Nestle U, Nevinny-Stickel M, Semrau S, Sterzing F, Wittig A, Andratschke N: Safety and efficacy of stereotactic body radiotherapy for stage 1 non-small-cell lung cancer in routine clinical practice: a patterns-of-care and outcome analysis. J Thorac Oncol 2013, 8:1050-1058.

19. Benedict SH, Cardinale RM, Wu Q, Benedict SH, Cardinale RM, Wu Q, Zwicker RD, Broaddus WC, Mohan R: Intensity-modulated stereotactic radiosurgery using dynamic micro-multileaf collimation. Int J Radiat Oncol Biol Phys 2001, 50:751-758.

20. Cardinale RM, Benedict SH, Wu Q, Cardinale RM, Benedict SH, Wu Q, Zwicker RD, Gaballa HE, Mohan R: A comparison of three stereotactic radiotherapy techniques; ARCS vs. noncoplanar fixed fields vs. intensity modulation. Int J Radiat Oncol Biol Phys 1998, 42:431-436.

21. Seco J, Sharp GC, Turcotte J, Seco J, Sharp GC, Turcotte J, Gierga D, Bortfeld $\mathrm{T}$, Paganetti $\mathrm{H}$ : Effects of organ motion on IMRT treatments with segments of few monitor units. Med Phys 2007, 34:923-934.

22. Dvorak P, Georg D, Bogner J, Dvorak P, Georg D, Bogner J, Kroupa B, Dieckmann K, Pötter R: Impact of IMRT and leaf width on stereotactic body radiotherapy of liver and lung lesions. Int I Radiat Oncol Biol Phys 2005, 61:1572-1581.

23. Hodge W, Tomé WA, Jaradat HA, Orton NP, Khuntia D, Traynor A, Weigel T, Mehta MP: Feasibility report of image guided stereotactic body radiotherapy (IG-SBRT) with tomotherapy for early stage medically inoperable lung cancer using extreme hypofractionation. Acta Oncol 2006, 45:890-896.

\section{doi:10.1186/s13014-014-0243-1}

Cite this article as: Yamashita et al:: Volumetric modulated arc therapy for lung stereotactic radiation therapy can achieve high local control rates. Radiation Oncology 2014 9:243.

\section{Submit your next manuscript to BioMed Central and take full advantage of:}

- Convenient online submission

- Thorough peer review

- No space constraints or color figure charges

- Immediate publication on acceptance

- Inclusion in PubMed, CAS, Scopus and Google Scholar

- Research which is freely available for redistribution 